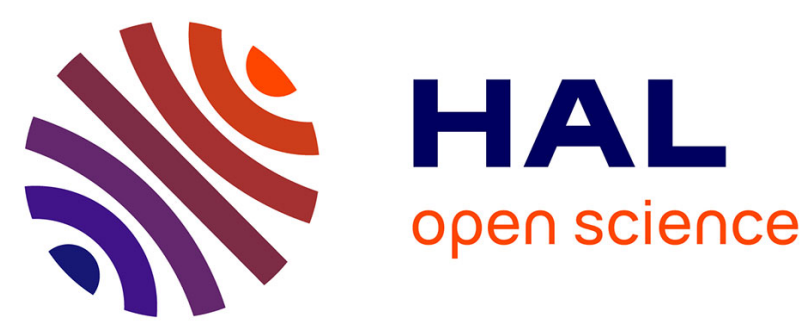

\title{
Les denrées végétales dans le nord de la Catalogne d'après les sources historiques et archéobotaniques (Xe-XVe siècles) : productions et échanges
}

\author{
Jérôme Ros, Carole Puig, Marie-Pierre Ruas
}

\section{- To cite this version:}

Jérôme Ros, Carole Puig, Marie-Pierre Ruas. Les denrées végétales dans le nord de la Catalogne d'après les sources historiques et archéobotaniques (Xe-XVe siècles) : productions et échanges. Archéologie du Midi Médiéval, 2014, 32, pp.123-138. hal-01613888

\section{HAL Id: hal-01613888 \\ https://hal.science/hal-01613888}

Submitted on 14 May 2020

HAL is a multi-disciplinary open access archive for the deposit and dissemination of scientific research documents, whether they are published or not. The documents may come from teaching and research institutions in France or abroad, or from public or private research centers.
L'archive ouverte pluridisciplinaire HAL, est destinée au dépôt et à la diffusion de documents scientifiques de niveau recherche, publiés ou non, émanant des établissements d'enseignement et de recherche français ou étrangers, des laboratoires publics ou privés. 


\title{
Pour citer cet article :
}

Ros J., Puig C. et Ruas M.-P. 2016, « Les denrées végétales dans le nord de la Catalogne d'après les sources historiques et archéobotaniques $\left(\mathrm{X}^{\mathrm{e}}-\mathrm{XV}^{\mathrm{e}}\right.$ siècles $)$ : productions et échanges », Archéologie du Midi Médiéval, Tome 32 - 2014 : 123-138.

\section{Les denrées végétales dans le nord de la Catalogne d'après les sources historiques et archéobotaniques $\left(\mathrm{X}^{\cdot-\mathrm{XV}} \mathrm{V}\right.$ - siècles) : productions et échanges}

\author{
Jérôme Ros ${ }^{*}$, Carole Puig ${ }^{* *}$, Marie-Pierre Ruas ${ }^{* * *}$
}

\section{Résumé (10 lignes)}

Situé à un carrefour d'influences culturelles entre les mondes méditerranéens de l'Espagne et du Midi de la France, le nord de la Catalogne (Pyrénées-Orientales, France), couvert par cette approche, est un site d'observation exceptionnel pour appréhender la circulation des produits, la transmission des techniques culturales et leur intégration dans les sociétés médiévales. La confrontation des données carpologiques issues de récentes synthèses avec celles des sources textuelles, notamment les tarifs de leudes, révèle aujourd'hui l'existence d'importants réseaux de commerce de denrées, depuis l'Espagne arabe jusqu'en Catalogne nord-pyrénéenne. A la lumière de ces différentes sources, on discutera des denrées végétales consommées dans cette région entre le $\mathrm{X}^{\mathrm{e}}$ et le $\mathrm{XV}^{\mathrm{e}}$ siècle, ainsi que de l'origine locale ou commerciale de certaines d'entre elles (céréales, figues, raisins).

Mots-clés : plantes, alimentation, commerce, production, Moyen Âge, carpologie, Roussillon Keywords: Plants, Food, Trade, Production, Middle Ages, Archaeobotany, Southern France

\section{INTRODUCTION}

Les questions de la mise en valeur des terroirs, des productions végétales et de l'évolution du patrimoine vivrier médiéval dans le nord de la Catalogne étaient, jusqu'à présent, peu documentées malgré plusieurs travaux sur les sources écrites ayant abordé directement ce volet: les productions et denrées végétales relevées et commentées concernant soit l'ensemble de la Catalogne, soit la Cerdagne (Bonnassie 1975-1976, 1990 ; Rendu 1991). Depuis ces publications, les dépouillements d'autres textes ont permis de détailler les

\footnotetext{
* Chargé de recherche CNRS. Institut des Sciences de l'Evolution - Montpellier, Univ. Montpellier, CNRS, IRD, EPHE. Place Eugène Bataillon, 34095 Montpellier Cedex 5, France.jerome.ros@umontpellier.fr

${ }^{* *}$ Chercheur associé FRAMESPA, UMR 5136. c.puig@free.fr

*** Directrice de recherche CNRS, Archéozoologie, archéobotanique : Sociétés, pratiques et environnements (UMR 7209), Sorbonne Universités, Muséum national d'Histoire naturelle, CNRS, CP 56, 55 rue Buffon, 75005 Paris, France. ruas@mnhn.fr
} 
productions agricoles médiévales de la plaine roussillonnaise et de la Cerdagne en pointant les biais de cette documentation (Puig 2003, 2005a, 2005b, 2005-2006, 2012).

Par ailleurs, sur le terrain archéologique, des recherches portant sur les terroirs de culture et les productions végétales à partir des assemblages de semences (ou restes carpologiques) dans les niveaux d'activité et d'habitat en plaine et sur les reliefs se sont développées pendant les années 1986-2003 à la faveur des opérations d'archéologie préventive dans la vallée de l'Agly et d'un programme collectif de recherche sur la Cerdagne'. Mais les données sont demeurées dispersées et ponctuelles. La plaine roussillonnaise n'a longtemps bénéficié que de la publication des concentrations de semences découvertes dans les fosses du haut Moyen Âge à Ruscino (Alessandri 1995) ou d'identifications de vestiges souvent extraits à vue, signalées dans les synthèses (Ruas 1998, 2005 ; Ruas et al. 2006). En montagne, deux études à Enveig et Angoustrine ont documenté la période médiévale antérieure au $\mathrm{X}^{\mathrm{e}}$ siècle de la Cerdagne (Ruas 2003 ; Campmajo et al. 2007). Quant au Capcir, il n'est documenté que par une seule étude carpologique des niveaux du $\mathrm{XI}^{\mathrm{e}}-\mathrm{XIII}^{\mathrm{e}}$ siècle du château des Angles (Ruas et Rendu 2005). Cependant, depuis 2006, la réalisation d'analyses carpologiques lors d'opérations d'archéologie préventive sur le tracé ferroviaire $\mathrm{LGV}^{2}{ }^{2}$ et de deux mémoires universitaires qui ont augmenté le corpus de données sur les contextes ruraux pour une période allant de la République à la fin du Moyen Âge (Ruas 2005, 2009, 2010-2011, 2012 ; Ros 2010, 2013 ; Ros et Ruas 2014). Ces travaux ont abouti à l'élaboration d'une première trame archéobotanique du patrimoine agro-alimentaire en Roussillon.

Alors que les données carpologiques sont désormais renforcées pour le haut Moyen Âge, particulièrement en Roussillon, il est apparu plus pertinent de cibler la présente approche sur la deuxième moitié du Moyen $\hat{A} g e\left(\mathrm{X}^{\mathrm{e}}-\mathrm{XV}^{\mathrm{e}}\right.$ siècle $)$ plus pourvue en textes qui autorise ainsi à croiser les deux types de sources, écrites et carpologiques. La limite géographique du corpus retenu comprend non seulement les régions rattachées alors à la Catalogne (plaine du Roussillon, reliefs du Vallespir, du Conflent des Aspres, du Capcir et de la Cerdagne), mais aussi le Fenouillèdes, qui, durant cette phase, était en partie placé sous autorité catalanoaragonaise (fig. 1).

L'objectif de cet article est de proposer une première image de l'éventail des denrées végétales produites, commercialisées et consommées dans l'extrémité nord de la Catalogne au cours de la période médiévale. Un croisement des données carpologiques et des sources

\footnotetext{
1 PCR transfrontalier 2001-2003 «Estivage et structuration sociale d'un espace montagnard: la Cerdagne», SRA Languedoc-Roussillon (coordination C. Rendu CNRS UMR 5136 FRAMESPA, Toulouse). Opérations archéologiques sur l'emprise du barrage de l'Agly (Caramany) 1986-1994

${ }^{2}$ Liaison ferroviaire Perpignan - Le Perthus (resp. J. Kotarba, INRAP périodes médiévales)
} 
textuelles sera tenté pour la première fois dans cette région afin d'identifier les plantes et les produits, les lieux de production ainsi que les réseaux commerciaux.

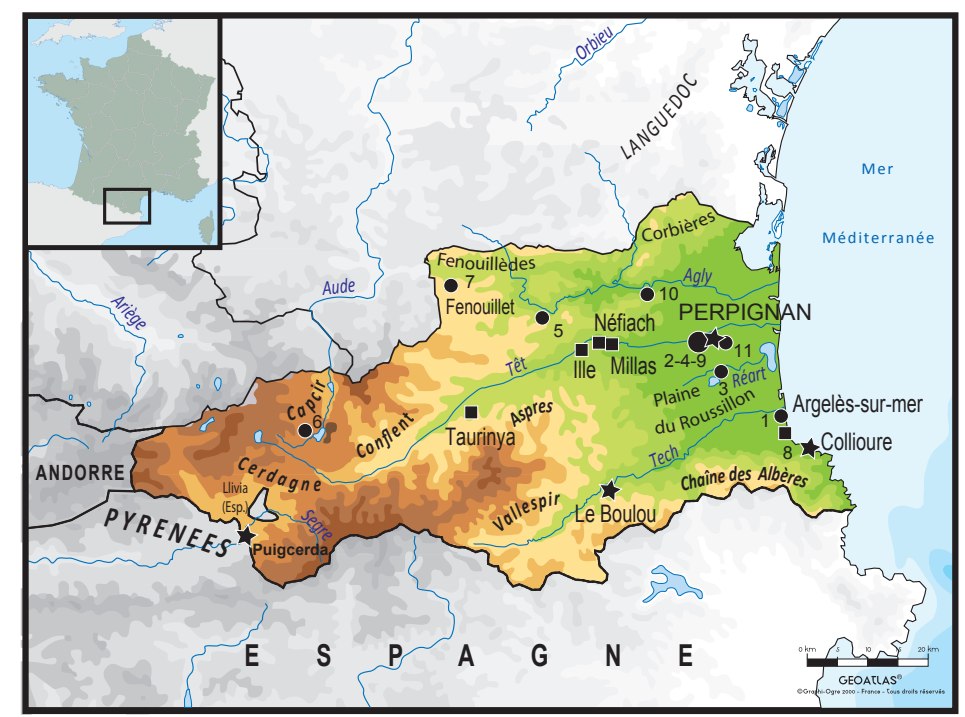

Fig.1 : Aire d'étude et localisation des sources carpologiques et textuelles citées dans le texte Symboles : ronds $=$ sites archéologiques (cf. Fig. 2), carrés = capbreus et Actes de la pratique, étoiles = leudes

\section{SOURCES ET MÉTHODES}

Les données archivistiques qui couvrent la période $\mathrm{du} \mathrm{X}^{\mathrm{e}}$ au $\mathrm{XV}^{\mathrm{e}}$ siècle ont été réunies dans le cadre d'un doctorat (Puig 2003) puis enrichies par plusieurs travaux postérieurs (Puig 2005a, 2005b, 2006, 2012). Elles émanent de fonds divers : actes de la pratique, ordonnances royales, archives commerciales ou encore recensements terriers. Ces deux derniers types de sources sont les plus instructifs pour éclairer le propos. En premier lieu, les tarifs de leudes (Gual Camarena 1968) ${ }^{3}$ permettent d'avoir une vision diversifiée des produits agricoles vendus sur les marchés roussillonnais et cerdans. Ils se présentent sous la forme d'une énumération de marchandises distinctes. Ce sont des sources uniques et d'une grande richesse pour ce type d'étude qui ont cependant de nombreux biais : l'origine des marchandises est rarement indiquée et le recensement des produits est partiel car ils ne sont spécifiés que lorsqu'ils sont taxés. Enfin, la dénomination des plantes et des denrées est souvent imprécise ou ambiguë

\footnotetext{
${ }^{3}$ Sources : Leudaire de Collioure, 1252, ACA, Batllia General de Catalunya, Tarifa de la leuda de Coplliure, $\mathrm{n}^{\circ} 450, \mathrm{f}^{\circ} 1$ à 6 et 65 à 71. Leudaire de Collioure 1298. ADPO 1B69 fo7-12. Leude de terre de Collioure, 1300, ADPO 1B69, f ${ }^{\circ} 4 \mathrm{r}-5 v^{\circ}$. Leudaire de Perpignan, ADPO 112 EDT3 Livre Vert Mineur de Perpignan, $\mathrm{f}^{\circ}$ 79-82 et $\mathrm{f}^{\circ}$ 82-85. Addenda au leudaire de

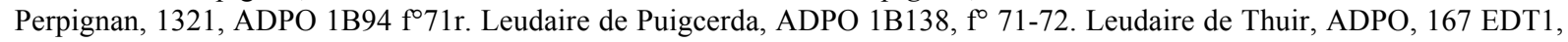
Livre Vert Mineur de Thuir, $\mathrm{f}^{\circ} 11$ et $\mathrm{f}^{\circ} 19 \mathrm{r}^{\circ}-20 \mathrm{r}^{\circ}$. Leudaire du Boulou, ADPO $1 \mathrm{~B} 94 \mathrm{f}^{\circ} 51$.

Gual Camarena (M.), Vocabularie del comercio medieval. Collección de aranceles aduaneros de la Corona de Aragón (Siglos XIII - XIV), Publicaciones de la Excelentìssima Diputación Provincial, Tarragone, 1968.
} 
(Puig 2005b). En second lieu, les terriers catalans ou capbreus ${ }^{4}$ indiquent plus sûrement des productions locales, mais les variétés végétales y sont rarement précisées. Elles sont perceptibles d'après la nature de la taxe qui pèse sur la parcelle (droit en céréales, en légumes, en fruits...) ou le type de parcelle (vigne, champ, jardins...). La documentation archivistique existante est cependant insuffisante pour établir une liste exhaustive de plantes ou de produits cultivés et/ou consommés dans le Roussillon médiéval. Cette démarche nécessite le brassage d'un grand nombre de sources de natures différentes, qui fournissent, au hasard des textes, des informations parfois ténues, souvent partielles.

Le corpus carpologique retenu pour cette étude est constitué de onze sites aux statuts variés (habitats ruraux, châteaux), implantés aussi bien en plaine roussillonnaise que sur les piémonts (Fenouillèdes) ou en montagne (Capcir) (fig.1, fig.2). Les restes végétaux proviennent de prélèvements de sédiment réalisés par les équipes de fouilles dans des contextes de nature différente mais appartenant à des zones d'habitats ou d'activités agricoles: foyers, fosses, puits, trous de poteau et niveaux d'abandon. Les méthodes d'extraction des vestiges archéobotaniques n'ont pas toujours été adaptées au matériel recherché en raison, pour les fouilles anciennes, de l'absence de protocole destiné spécifiquement à l'étude des semences. En effet, certains tamisages ont été effectués sous eau au travers de mailles comprises entre 2 et $6 \mathrm{~mm}$ de diamètre, les charbons de bois et certaines semences étant alors collectés à l'œil nu directement à partir des refus de tamisage par les équipes de fouilles. Cette méthode, peu efficiente pour repérer les semences de taille et de forme très diverses, a induit plusieurs biais dans la représentativité de ces assemblages carpologiques susceptibles d'être conservés (Marinval 1999). La majeure partie des échantillons a tout de même fait l'objet d'un tamisage par flottation sur mailles fines $(0,5$ et 2 $\mathrm{mm})$. Aussi, cette approche synthétique souffre-t-elle de l'absence d'homogénéité des méthodes d'échantillonnage et de la représentativité variable des données. Les semences extraites des sites de ce corpus sont conservées par carbonisation. Or, en raison de la forme de consommation des plantes (fraîche, broyée, grillée, bouillie...), des parties consommées (fruits, graines, feuilles, tiges, racines) et de leur probabilité d'être carbonisées lors d'accident ou d'opérations de transformations, les études carpologiques ne portent, la plupart du temps, que sur les graines et les fruits et d'autres macro-restes comme les parties végétatives que sont les bulbes, tubercules ou rhizomes qui ont pu résister à la combustion. Ainsi, la catégorie des

\footnotetext{
${ }^{4}$ Les registres sont conservés aux ADPO sous les cotes : Capbreu d'Argelès 1B30 (2Mi1/2), Capbreu de Collioure 1B29 (2Mi1/1), Capbreu d'Estagel 1B32 (2Mi1/4), Capbreu de Millas-Clayra 1B34 (2Mi1/6), Capbreu de Saint-Laurent 1B33 (2Mi1/5), Capbreu de Tautavel 1B31 (2Mi1/3).
} 
légumes à feuilles ou à racines (légumes verts) est-elle rarement représentée dans les spectres archéobotaniques (chou, poireau, épinard, salades par exemple), sauf sous forme des graines qui n'ont pas forcément été consommées ou qui le sont pour d'autres emplois.

Au-delà des limites inhérentes à chacun des corpus et aux méthodes d'analyses, la lecture croisée des données des sources écrites et carpologiques se heurte aussi à la difficulté de comparer les listes de plantes établies à partir d'identifications botaniques souvent précisées au rang de l'espèce, voire de la variété : Triticum aestivum (blé tendre), Hordeum vulgare (orge vêtue), Vicia faba var. minor (féverole) et des recensements des termes dans des textes qui désignent les plantes dans le langage vernaculaire régional médiéval. Ces noms en latin, parfois en catalan médiéval, sont clairement identifiables pour certains (orge/hordeus), beaucoup moins pour d'autres (citrullis, grau, bladum...). D'autre part, les sources écrites ne renseignent pas sur la variété culturale ; ainsi dans l'exemple de l'orge, la carpologie distingue les variétés vêtues et nues et les sous-espèces à deux ou six rangs tandis que les textes n'utilisent qu'un seul terme dont on ne sait pas s'il désigne toujours la même sorte d'orge. Aussi, l'identification de la plante, du produit ou du mets à partir de ces deux sources se heurte-t-elle au problème de la correspondance entre le nom botanique attribué à un reste végétal et le mot qui désigne un produit ou un mets dans les textes médiévaux qui utilisent un langage scientifique ou vernaculaire daté. Nous proposons donc les correspondances les plus probables déduites des données historiques et archéobotaniques pour chacune des plantes consignées dans les figures 3, 4, 5, 6 et 7

Les comparaisons terme à terme serviront ainsi à constater la présence ou l'absence d'une plante/denrée dans les listes élaborées à partir de ces deux sources. Par ailleurs, les paramètres d'évaluation du statut et de l'importance des plantes et des produits végétaux diffèrent entre les deux types de données. Mais par la confrontation des spectres de plantes ou de denrées, il est possible de repérer les productions locales du Roussillon, d'en caractériser le fonds agro-alimentaire et les plantes alimentaires secondaires, les nouvelles denrées végétales et/ou les plantes nouvelles apportées et adoptées au gré des contacts culturels et économiques. Dans cet article, les tableaux ne présenteront que les denrées attestées entre le $\mathrm{X}^{\mathrm{e}}$ et le $\mathrm{XV}^{\mathrm{e}}$ siècle. Mais les attestations connues dans les régions voisines ou en Roussillon aux époques antérieures seront signalées dans le texte. 


\begin{tabular}{|l|l|l|l|l|l|l|}
\hline Sites (Commune) & Localisation & $\begin{array}{l}\text { Datation } \\
\text { (siècles) }\end{array}$ & Responsable opération & $\begin{array}{l}\text { Nombre de } \\
\text { contextes }\end{array}$ & $\begin{array}{l}\text { Auteur analyse carpologique et } \\
\text { références bibliographiques }\end{array}$ & $\begin{array}{l}\text { Numérotation dans la } \\
\text { figure 1b }\end{array}$ \\
\hline L'Orangeraie (Taxo-d'avall) & Roussillon & IX-XII & N. Guinaudeau & 5 & 1 \\
\hline Mas de la Madeleine (Perpignan) & Roussillon & X-XI & J. Kotarba & 1 & Ros 2014 \\
\hline Saint Julien (Villeneuve-de-la-Raho) & Roussillon & X-XII & P. Alessandri & 1 & Ruas 1998, Ros 2013 \\
\hline Vilarnau d'Amont (Perpignan) & Roussillon & X-XII & O. Passarius & 1 & Ros 2013 \\
\hline L'Horto (Caramany) & Fenouillèdes & X-XII & A. Pezin & 10 & Ruas inédit dans Ros 2013 \\
\hline Château 2 castrum (Les Angles) & Capcir & XI-XIII & A. Bergeret & 5 & Ruas 2004 Ruas \& Rendu 2005 \\
\hline Château (Saint-Pierre de Fenouillet) & Fenouillèdes & XI-XIV & D. Maso & 6 \\
\hline Château royal (Collioure) & Roussillon & XII-XIV & O. Passarius & 3 & Hallavant 2009 \\
\hline Orle ouest (Perpignan) & Roussillon & XIII-XV & A. Bergeret & 1 & Ros et Ruas 2012 \\
\hline Camp del Rey (Baixas) & Roussillon & XX-XVI & O. Passarius & 6 & Ros 2013 \\
\hline Carrer del Farré (Cabestany) & Roussillon & XIV-XV & J. Kotarba & 1 & Ros 2013 \\
\hline
\end{tabular}

Fig. 2 : Corpus des données carpologiques (Pyrénées-Orientales)

\section{LES DENRÉES VÉGÉTALES}

Les listes de plantes alimentaires dressées ne peuvent être intelligibles pour former la base de la discussion que si les noms et les mots sont classés selon une nomenclature, en l'occurrence celle de l'alimentation ${ }^{5}$. Différents organes des plantes alimentaires sont consommés racines, tubercules, rhizomes, feuilles, tiges, bourgeons, bulbes, fleurs, graines et fruits - selon les espèces et les habitudes culturelles et religieuses. Or, les organes consommés peuvent entrer dans différentes catégories définies par les systèmes alimentaires et les valeurs culturelles et sociales accordées par la société (Bois 1927 ; Maurizio 1932 ; Braudel 1961 ; Grieco 1987, 1996). La diététique actuelle occidentale définit des catégories alimentaires classant ces organes selon les nutriments principaux qui les composent, les modes et formes de consommation et leur place dans les repas (Haudricourt et Hédin 1943 rééd. 1987). Dans l'inventaire, nous reprenons les catégories suivantes :

- légumes secs et féculents : céréales, légumineuses mûres, fruits riches en amidon comme les glands et les châtaignes, tubercules farineux, et, par extension, produits qui en sont tirés comme les pâtes ou le pain ;

- légumes verts ou légumes au sens large : racines (carotte, betterave), feuilles et tiges (chou, épinard, salade, cardes) et légumes fruits (concombre, gourde). Nous y ajoutons les bulbes des oignons et de l'ail qui, dans les énumérations médiévales, apparaissent comme légume ;

- aromatiques (herbes), épices et condiments ; organes divers à saveur aromatique (rhizome de gingembre, boutons floraux de la câpre ou du clou de girofle, pistil du safran, graines de fenouil, graines de moutarde, fruit de l'olive, feuille de menthe etc.),

- plantes oléagineuses: groupe des graines ou fruits riches en huile (lin, chanvre, pavot œillette, noix, noisette, amande, olive...). Dans ce groupe figurent des espèces dont les fibres des tiges sont aussi employées, mais pour des usages non alimentaires : le lin et le chanvre en particulier. En général, et par convention archéobotanique, elles sont réunies dans le groupe

\footnotetext{
${ }^{5} \mathrm{~F}$. Sigaut propose l'utilisation d'une nomenclature technique des termes référant à l'action, l'opération, le produit et le mets dans la transformation et la consommation des plantes. Produit (farine) et mets (semoule) sont donc différenciés (Sigaut 2010).
} 
mixte plantes techniques/oléagineuses pour marquer l'indécision quant à l'emploi de ces plantes lorsque des graines sont les seuls restes découverts ;

- fruits : fruits à coque et fruits charnus ou à pulpe. Notons qu'on utilise aussi le terme de fruit sec selon l'état dans lequel est consommé le produit et sa nature farineuse (voir féculent). Des fruits charnus et à coque y sont réunis : figue, raisin, datte, mais aussi noisette, amande ou noix ainsi que les fruits farineux (gland et châtaigne).

Ainsi, selon l'organe consommé, la substance utilisée, la façon de consommer et l'état de maturité, une même plante peut être rangée dans plusieurs catégories. Le carpologue recourt à des critères tels que le contexte de découvertes des restes et leur état (fossilisation et fragmentation) et leur abondance, les données archéobotaniques ou textuelles connues et ethnobotaniques pour classer l'espèce dans une catégorie (cas du lin ou du chanvre). Certains fruits sauvages attestés par la carpologie sont aussi inclus dans cette revue. D’autres éléments sont nécessaires pour interpréter ces résidus, à moins que le contexte n'éclaire le mode de préparation et/ou de consommation. Il ne peut satisfaire non plus complètement l'historien des textes confrontés à d'autres codes alimentaires et classements des denrées (cas de l'ail).

Pour discuter de denrées végétales et croiser les données, il faut donc prendre en compte à la fois la partie végétale citée et la façon dont les produits sont classés dans les textes consultés. Nous avons pris le parti d'utiliser la convention actuelle décrite-ci-dessus qui classe les plantes et les produits des deux corpus en gardant à l'esprit le caractère fixiste des catégories. Nous distinguerons, en outre, un groupe de fruits exotiques documentés sous ce statut par la documentation écrite.

La figure 3 indique les occurrences carpologiques des différents taxons mentionnés par site. Dans les figures $(4,6,7$ et 8$)$, les deux premières colonnes indiquent les mentions carpologiques obtenues à partir de l'identification des restes de plantes en précisant les noms latin et français du taxon, utilisés dans la nomenclature botanique (selon la base inventaire de l'INPN) ${ }^{6}$, mais aussi des conventions archéobotaniques (Jacomet 2006). Les deux autres colonnes consignent les mentions issues de l'analyse archivistique avec la traduction proposée pour le terme utilisé dans le texte. La lecture de gauche à droite suggère, à chaque ligne du tableau, la correspondance la plus probable entre le taxon botanique et le terme des textes et sa traduction.

\footnotetext{
${ }^{6}$ INPN, inventaire national du patrimoine naturel, Muséum national d'Histoire naturelle de Paris.
} 
L'ensemble des sources convoquées atteste soixante-quatre plantes alimentaires en Catalogne septentrionale entre le $\mathrm{X}^{\mathrm{e}}$ et le $\mathrm{XV}^{\mathrm{e}}$ siècle : quinze légumes secs, dont sept céréales et un terme non identifié, six ou sept légumineuses, quatorze légumes au sens large, vingt-trois fruits et douze épices/aromates.

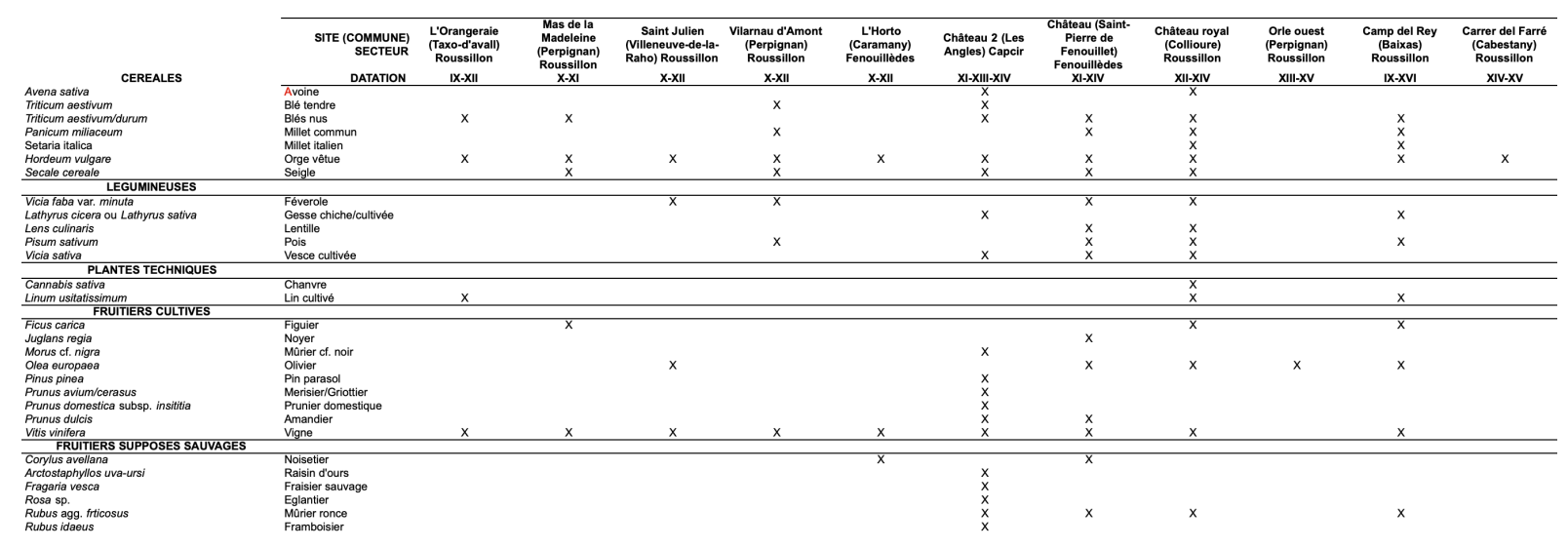

Fig.3 : Occurrences carpologiques des taxons par site $(\mathrm{X}=$ présence du taxon $)$

\section{Légumes secs à grains}

\section{Céréales}

Les céréales sont les plus fréquentes en contexte archéologique. Cette caractéristique tient à la fois à leur importance dans l'économie et l'alimentation des populations et aux modes de traitement que subissent les récoltes et les grains lors des nettoyages, de leur stockage et des préparations culinaires, qui favorisent leur état carbonisé et leur conservation dans les sédiments des sites aérobies (non immergés).

La correspondance entre les céréales identifiées dans les textes et les espèces enregistrées dans les dépôts archéologiques n'est pas systématique (fig.4). Dans le cas du blé nu, par exemple, son identification spécifique avec les restes carpologiques nécessite l'existence d'entre-nœud de rachis pour distinguer les trois espèces de blé nu possibles : le blé tendre (Triticum aestivum), le blé poulard (T. turgidum) et le blé dur (Triticum durum) (Jacomet 2006). Seuls les sites de Vilarnau d'Amont et du castrum des Angles ont livré des articles de rachis permettant d'attester un blé nu hexaploïde ou blé tendre (Triticum aestivum), à savoir le froment stricto sensu.

L'orge vêtue est présente dans dix des onze sites du corpus et le blé nu dans sept, toujours de façon dominante dans les assemblages dans lesquels ils sont identifiés (Ros 2010, 2013). Ils sont suivis par le seigle (cinq attestations) et le millet commun (quatre 
attestations). Les mentions de millet italien et d'avoine sont plus anecdotiques : la première est enregistrée uniquement sur les sites du château royal de Collioure (XII ${ }^{\mathrm{e}}-\mathrm{XIII}{ }^{\mathrm{e}}$ siècle) et du Camp del Rey $\left(\mathrm{XV}^{\mathrm{e}}-\mathrm{XVI}^{\mathrm{e}}\right.$ siècle), la deuxième sur ceux du château de Collioure (XII ${ }^{\mathrm{e}}$ $\mathrm{XIII}^{\mathrm{e}}$ siècle) (Ros 2013) et du castrum des Angles (XI ${ }^{\mathrm{e}}-\mathrm{XIII}^{\mathrm{e}}$ siècle) (Ruas et Rendu 2005). Un seul blé vêtu est enregistré, au château des Angles, sans qu'on puisse déterminer s'il s'agit d'amidonnier, d'engrain ou d'épeautre (Triticum type dicoccum/monococcum/spelta) (Ruas et Rendu 2005).

\begin{tabular}{|c|c|c|c|}
\hline \multicolumn{4}{|c|}{ CEREALES } \\
\hline \multicolumn{2}{|c|}{ Identifications carpologiques } & \multicolumn{2}{|r|}{ Mentions textuelles } \\
\hline Taxon latin & Taxon français & Terme usuel & Interprétation proposée \\
\hline Avena sativa & Avoine cultivée & Cibada, civada & Avoine \\
\hline Triticum aestivum & Blé nu hexaploïde (Blé tendre) & Forment, frumentus & Froment \\
\hline Panicum miliaceum et Setaria italica & Millet commun et Millet italien & Mill & Millets \\
\hline Hordeum vulgare & Orge vêtue & Ordeo & Orge \\
\hline \multicolumn{2}{|c|}{ aucune mention } & Aros/Arros & Riz \\
\hline Secale cereale & Seigle & Segle & Seigle \\
\hline \multicolumn{2}{|c|}{ aucune mention } & Grua & Céréale non identifiée \\
\hline \multicolumn{2}{|c|}{ aucune mention } & arraoni & Mélange d'orge et de froment (Méteil) \\
\hline
\end{tabular}

Fig.4 : Attestations des céréales d'après les sources carpologiques et textuelles

Dans les sources écrites, les céréales apparaissent communément sous l'appellation de bladum, qu'elles soient fourragères ou panifiables ${ }^{7}$. Une étude récente a permis de distinguer les céréales consommées par les hommes de celles destinées au fourrage (Puig 2012) (fig. 5).

\begin{tabular}{|c|c|c|c|c|}
\hline \multirow{2}{*}{ Céréales } & Céréales panifiables & \multirow{2}{*}{ Céréales commercées } & Alimentation animale \\
\cline { 2 - 3 } & Pain & Farine & $\mathrm{X}$ & \\
\hline Millet & & $\mathrm{X}$ & $\mathrm{X}$ & $\mathrm{X}$ \\
\hline Froment & $\mathrm{X}$ & $\mathrm{X}$ & $\mathrm{X}$ & $\mathrm{X}$ \\
\hline Avoine & & & $\mathrm{X}$ & \\
\hline Orge & $\mathrm{X}$ & $\mathrm{X}$ & $\mathrm{X}$ & \\
\hline Méteil & & $\mathrm{X}$ & $\mathrm{X}$ & \\
\hline Seigle & & & & Tous les mauvais grains \\
\hline Riz & & &
\end{tabular}

Fig.5 : Usages des céréales d'après les sources textuelles médiévales roussillonnaises (période Xe-XVe s. ) (d'après Puig 2012)

L'approche chronologique de la documentation archivistique permet également d'observer de nettes évolutions. Ainsi, les mentions les plus précoces font état de millet, de froment et d'avoine. Le millet, céréale peu référencée dans les sources roussillonnaises, fait partie des céréales les plus anciennes, clairement nommées dans la documentation à partir du $\mathrm{X}^{\mathrm{e}}$

\footnotetext{
${ }^{7}$ Alart, Cartulaire roussillonnais manuscrit, vol. XIV, p. 3, en 1342.
} 
siècle $^{8}$. Au XIII ${ }^{\mathrm{e}}$ siècle, il est parfois dit grosso sive tapato ou ergudelle, désignant peutêtre deux variétés à moins qu'il ne s'agisse de deux états dans son traitement ${ }^{9}$. A la fin du $\mathrm{XIII}^{\mathrm{e}}$ siècle, les sources écrites, par le biais des capbreus royaux de 1292-1293, révèlent une répartition géographique particulière. A Millas, en Ribéral, le millet est signalé par défaut car des redevances en céréales pèsent sur une terre mais ne doivent pas être versées en millet. Cette céréale est donc produite sur ce finage, mais elle n'est pas taxée. A l'inverse, le millet est très souvent mentionné dans le capbreu d'Argelès, sur le littoral, au même titre que l'orge et le froment. La production du millet en Roussillon nous apparaît ponctuellement mais le plus souvent en lien avec des terroirs humides, irrigués ou marécageux. Au XVII ${ }^{\mathrm{e}}$ siècle, les Roussillonnais le distinguent aussi du panis (Agusti, $\mathrm{f}^{\mathrm{o}} 200^{10}$ ), terme qui peut désigner le millet commun ou le millet italien, tous deux attestés par leurs grains au moins depuis le Bronze final dans cette région.

Le froment apparaît dans la documentation à partir du XI ${ }^{\mathrm{e}}$ siècle. Dans le courant du XII siècle, il est en retrait par rapport à l'orge car il est destiné à une population aisée ${ }^{11}$, et, par conséquent, il est moins référencé. Les occurrences augmentent ensuite à partir du XIV siècle et ne cesseront de croître pendant l'époque moderne. Cet essor est à mettre en relation avec le développement de la boulangerie en milieu urbain depuis le $\mathrm{XIV}^{\mathrm{e}}$ siècle (Puig 2012). L'avoine, comme le froment, se rencontre dans les sources médiévales roussillonnaises au $\mathrm{XI}^{\mathrm{e}}$ siècle, mais elle est très souvent associée à l'alimentation animale, et, plus précisément, au droit d'albergue $\mathrm{e}^{12}$.

A partir du XII ${ }^{\mathrm{e}}$ siècle, l'orge est la céréale la plus référencée dans la documentation. Elle sert à l'alimentation humaine mais aussi au fourrage (Puig 2003). Elle se maintient en Roussillon jusqu'à l'époque Moderne où le pain d'orge est alors réservé aux plus pauvres (Agusti, $\mathrm{f}^{\circ} 82 \mathrm{v}^{\circ}$ ). Un méteil apparaît aussi sous l'appellation d'arraoni. Celui-ci est un mélange d'orge et de froment ${ }^{13}$, mais dont on ne connaît pas les proportions en Roussillon. En Bas-Languedoc, la mescla ou le blé mitadenc, mentionnés dès le $\mathrm{XI}^{\mathrm{e}}$ siècle et en développement au XII ${ }^{\mathrm{e}}$ siècle, se composaient de deux parts de froment pour une part d'orge. Mais les proportions variaient selon le secteur (Durand 1991, 315).

\footnotetext{
${ }^{8}$ ADPO 12 J 25, 2 Mi 46 R11, Fonds Fossa ${ }^{\circ} 80$ / Cartulaire d'Elne $\mathrm{f}^{\circ} 137$. En 967, sous la forme « annone milium ».

${ }^{9}$ Alart, Cartulaire roussillonnais manuscrit, vol. XIV, p. 341 : En 1283 vente de 100 émines de millet dont 80 sont "de milio grosso sive tapato et residue 20 de milio ergudelle".

${ }_{10}^{10}$ Agusti (M.), Llibre dels secrets de agricultura, casa rùstica i pastoril, Barcelone, 1617, Barcelone, Alta Fulla, 1988.

11 Jusqu'au XIIIe siècle, en Roussillon, le froment semble réservé à une population privilégiée contrairement à l'orge qui constitue l'alimentation de base des roussillonnais (Puig 2003). A partir du XIIIe siècle, et en parallèle du développement de la boulangerie en ville, le pain de froment est se généralise en milieu urbain (Puig 2012).

${ }^{12}$ Sur les finages royaux, dont celui de Millas, droit comprenant l'hébergement d'un ou plusieurs soldats et la nourriture de sa monture. Capbreu de Millas.

13 1283, Alart, op. cit., vol. S, p. 338.
} 
Le seigle attesté dans les dépôts carpologiques du $\mathrm{X}^{\mathrm{e}}-\mathrm{XI}^{\mathrm{e}}$ siècle en montagne et en plaine (fig. 3) apparaît peu et assez tard dans les sources écrites roussillonnaises (fin XIII ${ }^{\mathrm{e}}$-XIV siècle). Cette céréale se rencontre surtout sur les piémonts roussillonnais, en Conflent et préférentiellement en Cerdagne. Le riz n'est pas exclu de la documentation roussillonnaise, puisqu'il est identifié dans les tarifs marchands ${ }^{14}$. Les sources font enfin mention d'une céréale au XIII ${ }^{\mathrm{e}}$ siècle, le grua indiquée comme une plante qui peut être cultivée entre deuX rangs de vigne, au même titre que d'autres céréales, légumes et légumineuses ${ }^{15}$. Elle est encore évoquée au XVII ${ }^{\mathrm{e}}$ siècle (Agusti fo 80 ), sans qu'il soit possible d'identifier la plante ou le produit auquel réfère ce terme.

Ainsi les céréales recensées dans les sources écrites comptent à la fois des espèces à grains nus (froment et seigle) et une majorité de céréales à grains vêtus (avoine, orge vêtue, millets, riz). Il est très probable que le terme ordeo réfère aux variétés d'orges vêtues devenues dominantes dans les dépôts carpologiques au détriment des variétés nues (Hordum vulgare var. nudum) en France et en Espagne depuis l'âge du Bronze (Buxó et Piqué 2008 ; Bouby 2010, 2014). Les sources écrites n’indiquent jamais si les céréales présentées sur les marchés sont décortiquées ou non. Toutefois, à partir du XIII ${ }^{\mathrm{e}}$ siècle, les actes de la pratique précisent que les grains composant la part seigneuriale doivent être boni et pulcri, signifiant qu'ils doivent être de bonne qualité et sain, mais peut-être aussi décortiqués et prêts à être stockés (Puig 2012). L'avoine et l'orge employées comme fourrage sont, en revanche, vraisemblablement fournies aux animaux sous forme de grains enserrés dans leurs balles. Mais, on doit se poser la question pour les grains d'orge qui servent aussi à confectionner les gruaux, les bouillies ou les pains et dont les grains enveloppés peuvent être moulus sous cette forme dans la mesure où la farine obtenue peut ensuite être tamisée. Les assemblages carpologiques livrent des grains dont la carbonisation a souvent fait disparaître les enveloppes. Blé nu et seigle, quant à eux, dont les grains sont libérés après un seul battage ou dépiquage, circulaient probablement à l'état de grains nus.

\section{Légumineuses}

Les découvertes carpologiques de légumineuses sont sporadiques et concernent généralement un faible nombre d'individus. Féverole, pois et gesses sont enregistrées dans

\footnotetext{
${ }^{14}$ Leudaire de Collioure, 1252, ACA, Battlia General de Catalunya, Tarifa de la leuda de Coplliure, $\mathrm{n}^{\circ} 450, \mathrm{f}^{\circ} 1$ à 6 et 65 à 71. Leudaire de Collioure 1298. ADPO 1B69 fo7 - 12. Leude de terre de Collioure, 1300, ADPO 1B69, $\mathrm{f}^{\circ} 4 \mathrm{r}^{\circ}-5 \mathrm{v}^{\circ}$.

${ }^{15}$ Coutumes de Perpignan, usages X.
} 
les sites ruraux de la plaine (Saint Julien, Vilarnau d'Amont, Camp del Rey) tandis que les sites castraux de plaine et de hauteur livrent plus de diversité car leur spectre comprend aussi des vesces et des lentilles. Les découvertes de ces graines en contexte archéologique sont trop ponctuelles pour évaluer pleinement leur importance dans les pratiques alimentaires et culturales médiévales de cette partie de la Catalogne. Sur les onze sites du corpus, seul le site du château 2 des Angles a livré une quantité significative de graines de gesse cultivée dans un foyer de bâtiment domestique daté des XII ${ }^{\mathrm{e}}-\mathrm{XIII}{ }^{\mathrm{e}}$ siècles mêlées à quelques graines de vesce (Vicia cf. sativa). En l'absence de féverole ou de pois, on peut se demander si ces gesses entraient dans l'alimentation des habitants ou celle du bétail. En effet, la palette végétale identifiée à partir des déchets des foyers de la basse-cour témoigne d'un accès à des denrées diverses dont plusieurs étaient obtenues à partir d'échanges avec les terroirs de la plaine (Ruas et Rendu 2005).

La culture de fèves et de pois est attestée par les sources écrites médiévales en Roussillon car elles apparaissent dans le paiement des redevances en nature dès le XII ${ }^{\mathrm{e}} \operatorname{siècle}^{16}$ (fig.6). $\mathrm{Au} \mathrm{XIII}{ }^{\mathrm{e}}$ siècle, les vesces sont à leur tour documentées ${ }^{17}$. Les tarifs marchands de l'intérieur des terres (Perpignan et Thuir) n'indiquent que la fève et le pois chiche, alors que ceux de Collioure, grand port du Roussillon, affichent une gamme plus diversifiée de légumineuses : pois chiche, gesse, pois, fève, lentille et vesce. Une seule mention confirme qu'elles sont en partie cultivées dans les jardins roussillonnais de Saint-Féliu d'Amont (fèves et pois) ${ }^{18}$.

\begin{tabular}{|l|l|l|l|}
\hline \multicolumn{3}{|c|}{ LEGUMINEUSES } \\
\hline \multicolumn{1}{|c|}{ Identifications carpologiques } & \multicolumn{2}{c|}{ Mentions textuelles } \\
\hline \multicolumn{1}{|c|}{ Taxon fatin } & \multicolumn{1}{|c|}{ Terançais } & Interprétation proposée \\
\hline Vicia faba var. minuta & Féverole & Faves & Fève ou Féverole \\
\hline Lathyrus cicera ou Lathyrus sativus & Gesse chiche ou Gesse cultivée & Guixes & Gesse \\
\hline Lens culinaris & Lentille & Lentilles & Lentille \\
\hline Pisum sativum & Pesols & Pois \\
\hline \multicolumn{2}{|c|}{ Pois cultivé } & Ciuros & Pois chiche \\
\hline Vicia sativa & Vesses/Besa & Vesce \\
\hline
\end{tabular}

Fig.6 : Attestations des légumineuses en Roussillon d'après les sources carpologiques et textuelles (période Xe-XVe s. )

Compte tenu des mentions écrites, il est troublant que les sites archéologiques du Roussillon n'aient pas livré de pois chiches alors qu'ils sont attestés dans sept sites en Languedoc et en Provence entre le VIII ${ }^{\mathrm{e}}$ et le $\mathrm{XVI}^{\mathrm{e}}$ siècle. En Languedoc, ils se manifestent dans deux sites de la plaine alluviale à Lunel-Viel (Hérault) pour le $\mathrm{X}^{\mathrm{e}}-\mathrm{XI}^{\mathrm{e}}$ siècle (Ruas

\footnotetext{
${ }^{16}$ ADPO 12 J 25, 2 Mi 46 R11, Fonds Fossa n ${ }^{\circ} 175$ / Cartulaire d'Elne $\mathrm{f}^{\circ} 88 \mathrm{v}^{\circ}$, en 1128.

${ }_{17}^{17}$ Alart, op. cit. vol. S, p. 338, en 1283.

${ }^{18}$ Alart, op. cit. vol. VII, p. 610, ADPO Cartulaire du Temple, $\mathrm{f}^{\circ} 96 \mathrm{r}^{\circ}$, en 1180.
} 
1990), à Plaissan les Termes (Hérault) daté du $\mathrm{IX}^{\mathrm{e}}-\mathrm{X}^{\mathrm{e}}$ siècle (Ruas 2005) et dans deux sites de la moyenne montagne à Durfort (Tarn) à la fin du XIII ${ }^{\mathrm{e}}$ siècle (Ruas 1998, 2002) et à La Cisterne à Cabrières (Hérault) (Ruas 1998). En Provence, ils sont attestés dans trois sites à Augéry-de-Corrèges (Bouches-du-Rhône) pour le $\mathrm{VIII}^{\mathrm{e}}-\mathrm{X}^{\mathrm{e}}$ siècle (Ruas 1998, 2005) et dans l'arrière-pays, à Vauvenargues (Bouby 1996 inédit) et Allemagne-en-Provence dans les Alpes-de-Haute-Provence (Ruas 2015), occupations du XI ${ }^{\mathrm{e}}$ siècle. Légumineuse du fonds alimentaire méditerranéen, ses mentions carpologiques médiévales demeurent curieusement rares ou localisées.

La vesce se manifeste à Collioure dans les sources écrites ${ }^{19}$ et un dépôt du Château. La mention de cette graine dont l'espèce n'est pas confirmée dans un foyer du castrum des Angles en Capcir est trop occasionnelle pour en déduire sa culture en montagne. Toutefois, le foyer de Montaillou en Ariège, daté de la fin du XII ${ }^{\mathrm{e}}-\mathrm{XIII}^{\mathrm{e}}$ siècle a livré une abondance de vesce qui témoigne d'une réelle culture de cette légumineuse dans les terroirs de haute montagne pyrénéenne (Hallavant et Ruas 2007). Alors que ses occurrences sont élevées dans les sites du haut Moyen Âge du nord de Paris et qu'elle n'apparaissait pas dans les sites méridionaux (Ruas 2005, 2011), les attestations de la vesce se multiplient ces dernières années dans les sites du sud de la France à la faveur de l'augmentation des séries carpologiques étudiées (Ros 2013).

\section{Légumes verts}

Pour ce qui concerne les parties végétatives en général récoltées et consommées avant la formation des fruits, elles sont rarement enregistrées dans les sites. Le corpus carpologique commenté dans cet article ne dispose d'aucun contexte archéologique immergé (de type puits ou latrine urbaine), aussi les légumes verts sont-ils absents des spectres (fig.7).Toutefois, des espèces de cette catégorie ont pu être attestées par des graines hors de l'aire d'étude : l'épinard (Spinacia oleracea) à Montaillou à la fin du XII ${ }^{\mathrm{e}}-\mathrm{XIII}{ }^{\mathrm{e}}$ siècle (Hallavant et Ruas 2008), le cresson alénois (Lepidium sativum), le poireau (Allium porrum), l'amaranthe blite (Amaranthus blitum), la gourde (Lagenaria siceraria) dans une latrine de Montauban (Tarn-et-Garonne) du XIV ${ }^{\mathrm{e}}$ siècle (Ruas 1998, 2008, 122) et de probables bulbes d'ail/oignon (cf. Allium) dans les niveaux incendiés du X $\mathrm{X}^{\mathrm{e}}-\mathrm{XI}^{\mathrm{e}}$ siècle de la Moutte à Allemagne-en-Provence (Ruas 2015) et du XIV siècle du castrum de la Muggliunaccia à Olmi-Capella (Haute-Corse) (Ruas 1998).

\footnotetext{
${ }^{19}$ Alart, op. cit., vol. S, p. 338 en 1283.
} 
Dans l'état actuel des recherches carpologiques en Roussillon, seuls les textes renseignent sur cette catégorie alimentaire. Le paysan roussillonnais cultive dans son jardin des poireaux et des choux (porris et caulibus), des carottes (pastanagarum), des oignons (ceparum) et des navets (nadui) ${ }^{20}$. Les gourdes (cucurbitum) et les concombres (cucumerorum) sont plus rares ${ }^{21}$. Avec les leudaires, la liste s'enrichit de l'ail, de la laitue, de l'épinard, de la bette, de l'aubergine et de l'échalote. La vente de graines (notamment en ce qui concerne l'aubergine, le concombre et la gourde calebasse) sur les marchés roussillonnais confirme que ces légumes étaient cultivés localement (Puig 2003) (fig.7).

\begin{tabular}{|c|c|c|c|}
\hline \multicolumn{4}{|c|}{ LEGUMES VERTS } \\
\hline \multicolumn{2}{|c|}{ Identifications carpologiques } & \multicolumn{2}{|c|}{ Mentions textuelles } \\
\hline Taxon latin & Taxon français & Terme usuel & Interprétation proposée \\
\hline \multirow{14}{*}{\multicolumn{2}{|c|}{ aucune mention }} & All/Almoll & Ail \\
\hline & & Albudeca & Aubergine \\
\hline & & Bettis & Bette \\
\hline & & \begin{tabular}{|l} 
Pastanagarum \\
\end{tabular} & Carotte \\
\hline & & Col/Coulibus & Chou \\
\hline & & Cucumerorum/Congombre & Concombre \\
\hline & & Scalunyes & Echalote \\
\hline & & Spinargiis & Epinard \\
\hline & & Cucurbitum & Gourde \\
\hline & & Cucurbitus/Carabasse & Gourde calebasse \\
\hline & & Lautucis & Laitue \\
\hline & & Naps & Navet \\
\hline & & Cebes/Ceparum & Oignon \\
\hline & & Porro/Porris & Poireau \\
\hline
\end{tabular}

Fig.7 : Attestations des légumes verts en Roussillon d'après les sources carpologiques et textuelles (période Xe-XVe s.)

\section{Fruits}

Les sources écrites témoignent d'une dichotomie en ce qui concerne les mentions de fruits (Puig 2006) (fig.8). Les actes de la pratique évoquent très peu les fruitiers, presque jamais les fruits, en dehors des essences «phares» comme la vigne et l'olivier. Pourtant les toponymes indiqués dans les actes médiévaux suggèrent la présence d'autres espèces : Castaines, Pererium, Pruner..., mais les fruits restent dans l'ombre et même le raisin (frais) n'est jamais évoqué dans ce type de sources. Les informations sont donc tirées essentiellement des leudaires.

\footnotetext{
${ }^{20}$ ADPO 1B35 en 1120 à Salses.

${ }^{21}$ Alart, op. cit., vol. XII, p. 53, en 1295.
} 


\begin{tabular}{|c|c|c|c|}
\hline \multicolumn{4}{|c|}{ FRUITS } \\
\hline \multicolumn{2}{|c|}{ Identifications carpologiques } & \multicolumn{2}{|c|}{ Mentions textuelles } \\
\hline Taxon latin & Taxon français et nom du fruit & Terme usuel & Interprétation proposée \\
\hline Arctostaphyllos uva-ursi & Raisin d'ours, busserole & \multicolumn{2}{|c|}{ aucune mention } \\
\hline Corylus avellana & Noisetier, noisette & Avenes/Avellans & Noisette \\
\hline Ficus carica & Figuier, figue & Figa & Figue \\
\hline Fragaria vesca & Fraisier sauvage, fraise des bois & \multicolumn{2}{|c|}{ aucune mention } \\
\hline Juglans regia & Noyer, noix & Nots & Noix \\
\hline Morus cf. nigra & Mûrier cf. noir, mûre noire & \multicolumn{2}{|c|}{ aucune mention } \\
\hline Olea europaea & Olivier, olive & Oliva & Olive \\
\hline Pinus pinea & Pin parasol, pignon & Pinyes & Pignon \\
\hline Prunus avium/cerasus & Merisier ou Griottier, cerise & Civeris & Cerise \\
\hline Prunus domestica subsp. insititia & Prunier crèque, prune & \multicolumn{2}{|c|}{ aucune mention } \\
\hline Prunus dulcis & Amandier, amande & Amelo & Amande \\
\hline Quercus sp. & Chêne, gland & Glan & Gland \\
\hline Rosa sp. & Eglantier, cynorrhodon & \multicolumn{2}{|c|}{ aucune mention } \\
\hline Rubus agg. Fructicosus & Ronce de Bertram, mûre de ronce & \multicolumn{2}{|c|}{ aucune mention } \\
\hline Rubus idaeus & Framboisier, framboise & \multicolumn{2}{|c|}{ aucune mention } \\
\hline Vitis vinifera & Vigne, raisin & Aztebib & Raisin \\
\hline \multirow{7}{*}{\multicolumn{2}{|c|}{ aucune mention }} & Castanyes & Châtaigne \\
\hline & & Citrullis & Pastèque \\
\hline & & Datils & Datte \\
\hline & & limons e pomers e terongers & Agrumes (plusieurs sortes) \\
\hline & & Melons & Melon \\
\hline & & Peres & Poire \\
\hline & & Pressec & Pêche \\
\hline
\end{tabular}

Fig.8 : Attestations des fruits en Roussillon d'après les sources carpologiques et textuelles (période Xe-XVe s.)

\section{Fruits à pulpe}

De nombreux fruits à pulpe sont indiqués à Narbonne en $1153^{22}$ et à Barcelone en $1222^{23}$, mais ils n'apparaissent pas dans la documentation roussillonnaise ou cerdane. Ils y sont peutêtre taxés sous la forme générique de «fruyta». Seules les cerises sont indiquées à Perpignan en 1250 , et, curieusement, poires et pêches à Puigcerda en $1288^{24}$. La présence de fruits en contexte urbain, et dont le commerce est inséré dans une dynamique marchande, n'est pas étonnante. En revanche, la présence de fruits aussi fragiles que la pêche à $1200 \mathrm{~m}$ d'altitude pose question. Faut-il envisager sa production locale ou sa vente sous une forme autorisant une meilleure conservation? Aucune découverte carpologique pour cette période ne vient éclairer la consommation de poire et de pêche dans cette partie de la Catalogne.

La vigne et l'olivier, plus que discrets dans les textes évoqués ici, sont les fruitiers les plus souvent attestés en contexte archéologique roussillonnais des périodes historiques (Ros 2010, 2013). La vigne est présente dans neuf des sites du corpus, l'olivier dans cinq.

Deux autres espèces cultivées de fruits charnus sont enregistrées par la carpologie : mûres noires, prunes et merises/ou griottes ont été découvertes dans le seul site du château 2 des Angles (Ruas et Rendu 2005), dans des contextes datés fin $\mathrm{XI}^{\mathrm{e}}$-début XIII ${ }^{\mathrm{e}}$ siècle. Les hasards de la documentation permettent quand même d'attester la culture du mûrier en Conflent, à

\footnotetext{
${ }^{22}$ Germain Mouynés (M.), Inventaire des archives communales antérieures à 1790, Emmanuel Caillard Imprimeur, Narbonne, 1871, Annexes de la Série AA, pp. 4 à 6.

${ }^{23}$ Gual Camarena (M.), Vocabularie del comercio medieval. Collección de aranceles aduaneros de la Corona de Aragón (Siglos XIII-XIV), Publicaciones de la Excelentìssima Diputación Provincial, Tarragone, 1968.

${ }^{24}$ ADPO, 1 B 138, fo $71-72$.
} 
Moligt. Ainsi en 1024, est-il fait mention de plusieurs maisons dotées de mûriers (jusqu'à trois arbres) plantés dans un jardin ${ }^{25}$. En revanche, d'autres fruits mentionnés par les textes, comme le melon et la pastèque ne se manifestent dans aucun site archéologique du corpus. Des mentions carpologiques de pépin de melon existent pourtant dans le sud de la Catalogne, à Lleida ( $\mathrm{XI}^{\mathrm{e}}$ siècle), en contexte imbibé ou minéralisé (Alonso 2005).

Les assemblages carpologiques, quant à eux, révèlent aussi des fruits de cueillette et enregistrent notamment des cynorrhodons (fruits de l'églantier), de la busserole (fruit du raisin d'ours), de la fraise des bois et de la mûre de ronce au château 2 des Angles, des framboises, des cynorrhodons et des mûres de ronce à Enveig (Ruas 1998, 2003). La mûre de ronce est attestée à la même période à Saint-Pierre de Fenouillet (Hallavant 2009), Collioure (Ros 2013) et au Camp del Rey (Ros 2013), faisant d'elle le troisième fruit le plus fréquent du corpus, après la vigne et l'olivier. La récolte locale de tels fruitiers sauvages dans le cadre d'activités domestiques explique leur absence des textes commerciaux ou des actes de la pratique.

\section{Fruits à coque}

Les fruits à coque sont assez bien documentés par les textes : les amandes, par exemple, sont vendues avec la coque ${ }^{26}$ (Puig 2006). À Perpignan, elles sont aussi associées au riz et supportent une taxe différente, ce qui pourrait signifier qu'elles sont alors vendues sans la coque. À Collioure en 1298, on vend des noisettes avec la coque, en sac, comme les châtaignes et les noix ${ }^{27}$. Le noyer est un des rares fruitiers roussillonnais à être indiqué dans les actes de la pratique ${ }^{28}$. Enfin, le pignon, également indiqué à Collioure, apparaît dans les actes de la pratique sous la forme d'un pinyer royal. Celui-ci désignerait un bois de pins parasol relevant de l'autorité royale (Puig 2006). Les fruits à coque mentionnés à Collioure ont deux origines possibles. Soit, ils sont issus de productions locales transportées depuis les coteaux des Albères proches, soit leur commerce découle d'importation à grande échelle, leur état sec naturel facilitant grandement leur conservation pendant le transport.

Les indices carpologiques de fruits «à coque» sont plus discrets. La noisette et la noix sont régulièrement attestées dans les sites roussillonnais (Ros 2010, 2013), mais se

\footnotetext{
${ }^{25}$ ADPO H142: "Et super ipsa casa orto I qui vocant Aspirio cum morarios III et trans ipsa casa de Mirone orto I cum ipso morario ».

${ }^{26}$ Leudaire de Perpignan de 1284 et de Collioure de 1365.

${ }^{27}$ Leudaire de Collioure, 1298.

${ }^{28}$ ADPO 1B35, en 1119.
} 
rencontrent préférentiellement dans les implantations de versants ou d'altitude. L'amande est attestée au château 2 des Angles (Ruas et Rendu 2005) et à Saint-Pierre de Fenouillet (Hallavant 2009). La rareté des coques de ce fruit en contexte archéologique pose la question de l'utilisation de ces éléments une fois la graine (amande) extraite. En effet, l'utilisation des coques du fruit comme combustible pourrait expliquer qu'on en retrouve si peu et sous une forme très fragmentée.

Les pignons et les glands, bien que connus dans la région dans des contextes altomédiévaux comme à Manresa et Ruscino (Ruas 2010-2011; Ros et Ruas 2014), sont rares dans les spectres plus tardifs ; seul le premier se manifeste par un fragment de bractée au château royal de Collioure (Ros 2013). Or, le leudaire du Boulou, village d'arrière-pays, présente la particularité de mentionner les glands de chêne au même titre que les céréales et les légumes. Ces glands vendus dans le village étaient certainement ramassés dans des chênaies locales ${ }^{29}$. Le rôle de ce fruit farineux demeure obscur mais les indices suggèrent son éventuel emploi régional dans l'alimentation humaine régionale (Puig 2006; Ros et Ruas 2014). La châtaigne n'est, quant à elle, attestée que dans un seul site médiéval du sud de la France, à Bayonne (Pyrénées-Atlantiques) dans les rebuts d'un égout urbain daté du $\mathrm{XII}^{\mathrm{e}}-\mathrm{XIV}^{\mathrm{e}}$ siècle (Ruas et al. 2006).

\section{Fruits exotiques et du commerce lointain}

Les marchés roussillonnais proposent d'autres sortes de fruits importés qui semblent inconnus ou qui diffèrent de la palette alimentaire habituelle. Certains sont exotiques (dattes, agrumes), d'autres (figue, raisin) probablement vendus séchés car leur origine est lointaine. Les attestations de la datte s'observent le long du littoral méditerranéen; elle était importée sous forme sèche ${ }^{30}$. L'origine précise du fruit n'est malheureusement pas connue. Le deuxième leudaire de Collioure se distingue par la mention d'agrumes : limons e pomers e terongers. Si les termes permettent d'identifier aisément le citron et l'orange, que désigne pomers? L'attribution de ce terme à la grenade a été envisagée, mais celle-ci semble apparaître plus généralement sous la forme granarios. Il semblerait ainsi que ce terme désigne un agrume, groupe auquel il est associé dans les énumérations. On peut penser au pamplemousse, fruit qui se serait répandu pendant la période islamique vers la Méditerranée occidentale (Watson 1983 réed 2008 ; Albertini 2013); le Roussillon correspondrait ainsi aux aires d'échanges des fruits présents sur le marché de Collioure.

\footnotetext{
${ }^{29}$ ADPO 12J25. Le chêne est dit glandiferis oglatis, en 972.

${ }^{30}$ Leudaires de Collioure.
} 
Néanmoins le cédrat, mais aussi la mandarine ou la bergamote ont pu circuler dans le Bassin Méditerranéen à cette date. Il semblerait que ces produits soient assez récents sur ce marché : le tarif de la leude qui les concerne est suivi de la phrase selon laquelle tous les produits qui "ne sont ni nommés ni connus des leuders [chargés de prélever la leude] valent selon les produits précédents ${ }^{\text {‘31 }}$. À cette date, il s'agirait donc de nouveautés, mais que l'on connaît suffisamment pour pouvoir les nommer (Puig 2005-2006) ${ }^{32}$.

La figue, bien qu'elle apparaisse peu dans la documentation, a fait l'objet d'une production locale difficile à évaluer ${ }^{33}$. Toutefois, les leudaires de Collioure font état de diverses figues, sans aucun doute séchées, originaires de Mallorque, Alicante, Tortose, Denia, Malaga, Valence, Tarragone ou Murcie. On note également dans les tarifs marchands que les taxes qui pèsent sur le panier de figues varient selon l'origine du fruit (Puig 2006). Le raisin se manifeste seulement sous la forme d'un terme andalou aztebib, qui désigne le raisin sec. Il est bien souvent taxé avec les figues et les dattes (Puig 2005-2006).

En l'état actuel des études, les fruits exotiques mentionnés dans les textes (dattes et agrumes) ne sont pas enregistrés par la carpologie dans la zone d'étude. Des « noyaux » de datte, fruit attesté dans les sites funéraires à la période romaine en France, ne sont enregistrés que dans trois sites médiévaux dans le nord du pays (Ruas et al. 2006 ; Ruas inédit). Pourtant, dans les traités culinaires français, leur chair est utilisée associée aux figues et aux raisins secs pour leur saveur sucrée (Mane 2006). Les figues et les raisins enregistrés par la carpologie ne sont jamais conservés entiers dans les sites médiévaux du Roussillon et ne sont présents que sous la forme des pépins. Or certains assemblages carbonisés des sites du haut Moyen Âge ont livré des restes de raisin composés de pépins, de quelques baies et pédicelles, interprétés comme des résidus de pressurage (Ros et al. accepté). Les assemblages tardifs pris en compte dans cet article, correspondraient davantage à des résidus de consommation, peut-être de raisin sec, qui évoqueraient alors les raisins secs importés (aztebib des textes) ou locaux.. La rareté des sous-produits de pressurage a été relevée dans les sites médiévaux du Midi de la France (Ruas et al. 2006) et soulevait la question des lieux d'exercice de cette activité qui, dans certaines régions, pourrait être localisés dans les vignes (Puig 2003).

\footnotetext{
${ }^{31}$ Leudaire de Collioure 1298.

${ }^{32}$ Ce qui favoriserait aussi l'interprétation de pomers comme un agrume et un fruit qui n'est pas originaire de Méditerranée nord-occidentale.

${ }^{33}$ ADPO 12J25, en 972.
} 


\section{Épices, aromates, condiments}

Outre le fait que les plantes de la catégorie épices/aromates/condiments présentent des organes variés peu propices à la conservation dans les sites archéologiques aérobies, celles dont on a utilisé les fruits et les graines sont souvent broyées dans les préparations culinaires (Laurioux 1983), notamment celles données par les traités culinaires occitans et catalans des $\mathrm{XIII}^{\mathrm{e}}$ et $\mathrm{XIV}^{\mathrm{e}}$ siècles (Lambert 1992). Par ailleurs, les semences de certaines espèces herbacées comme l'aneth ou la moutarde, peuvent aussi provenir de plantes sauvages mauvaises herbes des cultures. Les cueillettes peuvent toutefois concerner de telles plantes utilisées dans l'alimentation humaine. Une seule mention de graine minéralisée de moutarde blanche existe en Roussillon, dans un contexte du $\mathrm{VI}^{\mathrm{e}}$ siècle du site des Xinxetes (Saint-Cyprien) (Ros 2013). Une graine de galbule de genévrier (Juniperus communis) est attestée dans les niveaux de combustion du château 2 des Angles. Mêlée à d'abondantes semences de céréales et de légumineuses dans les cendres de foyer, cet unique individu laisse douter d'une collecte intentionnelle dans un but alimentaire aromatique. Il est plus probable que des rameaux fructifères aient servi de combustible et que des fruits aient brûlé à cette occasion.

Les sources écrites sont plus détaillées sur la question. En effet, les tarifs marchands renseignent relativement bien sur le commerce de condiments et aromates : des « herbes à cuire » (erba cucera) sont indiquées à Collioure et Perpignan, de l'anis ou aneth (comie aniç, annyins), de l'armoise, du serpolet (serpelers), du basilic (Alfarega), de la menthe (menta), de l'origan (auruga), de la réglisse (reglissia) et de la moutarde (mostasia). On y rencontre aussi des fleurs : roses (roses), violettes (violes), bourrache (borragiis), fleur de florinatie ( ?), de l'aubépine ou de la lavande (espic), du chardon ou de l'artichaut (flor de cardo). L'armoise, la menthe, le basilic et la bourrache sont indiquées dans les « Usages de Perpignan » datés des $\mathrm{XI}^{\mathrm{e}}$-XIII ${ }^{\mathrm{e}}$ siècles comme des plantes qui sont cultivées localement. En outre, une grande variété d'épices très certainement importées est également indiquée : safran, poivre, cannelle...

\section{CULTURE, CONSOMMATION, COMMERCE}

Le spectre végétal obtenu par la confrontation des données textuelles et carpologiques permet d'attester une large palette de plantes dont l'origine et les zones potentielles de mise en culture ne sont cependant pas toujours définies ou localisées. Par mise en culture, on entendra le fait de cultiver une espèce sans qu'elle soit nécessairement destinée au commerce; les assemblages carpologiques étudiés étant pour la plupart plus révélateurs d'activités 
quotidiennes souvent domestiques (sites ruraux modestes) que de pratiques culturales pour une production de rapport orientée vers la vente à grande échelle.

\section{Culture locale ?}

Les assemblages carpologiques et les textes s'accordent pour envisager la culture locale de certaines des espèces inventoriées, telles les céréales et les légumineuses. En contextes archéologiques, leurs semences sont, en effet, mêlées à des adventices de cultures dont l'écologie témoigne qu'elles proviennent de parcelles cultivées dans les milieux environnants les sites.

Pour les céréales, le blé nu et l'orge vêtue apparaissent comme les produits les plus courants. La culture des autres céréales n'est pas toujours avérée en plaine par les restes carpologiques ou sur les lieux mêmes de la mention textuelle.

Les rares mentions carpologiques du seigle en Roussillon s'ajoutent à celles des sites languedociens de Médor (Ornaisons, Aude) près de Narbonne et de la place Conesa à Agde $(\text { Hérault })^{34}$, datées du VI ${ }^{\mathrm{e}}$ siècle. L'abondance des rachis d'épi à Médor et des grains à Agde suggère qu'il y fut cultivé (Ruas $1989 ; 2005$ ). Sa culture est bien attestée en Conflent (Taurinya), en Ribéral (Ille, Néfiac) ou à Argelès, par les textes sans qu'il soit possible d'en évaluer l'importance (Puig 2003). Les assemblages carpologiques des Angles à 1600 m (Ruas et Rendu 2005) et les actes notariés de Puigcerda de la fin du XIII ${ }^{\mathrm{e}}$ siècle (Rendu 1991) témoignent de son exploitation en haute montagne capcinoise et cerdane. Les données carpologiques indiquent que le millet était communément semé en plaine durant la période alto-médiévale (Ros 2013). Les carporestes enregistrés aux périodes postérieures sont insuffisants pour témoigner d'une production significative. Sa culture à la fin du XIII ${ }^{\mathrm{e}}$ siècle est pourtant bien attestée par les Capbreus à Millas et à Argelès, au même titre que l'orge et le froment. Cette céréale apparaît ainsi dans la documentation sous la forme d'une taxe en nature.

Si les occurrences carpologiques d'avoine se multiplient au début du Moyen Âge en France (Ruas 2005), les petites quantités de grains découverts, même s’il s'agit d'Avena sativa, ne suggèrent pas toujours sa culture en Roussillon ; adventice commune des autres céréales, son statut demeure flou. Mais son exploitation dans la région est bien documentée par les textes, bien qu'elle semble plutôt destinée au bétail.

\footnotetext{
${ }^{34}$ Étude inédite de L. Bouby 2002, fouilles D. Ugolini
} 
Enfin, en l'état actuel des recherches, il existe très peu d'indices démontrant la culture du riz dans le nord de la Catalogne. Indiqué dans les deux leudes de Collioure, il aurait été mis en culture au $\mathrm{XV}^{\mathrm{e}}$ siècle dans la région de Perpignan, selon la proclamation du lieutenant du vice-roi [d'Aragon] « [...] vedelicet quod negun non habe a semenar aros una leuga entorn Perpinyan $»^{35}$ (Cheyronnaud 2006), indiquant que la culture du riz ne devait pas être pratiquée dans la périphérie proche de la ville. Il s'agirait-là de la plus ancienne mention de culture de cette céréale en France. Les céréales ont pu être cultivées en champ (irrigués ou non), qui auraient pu alors accueillir les légumineuses dont les attestations carpologiques sont variables.

Les textes apportent quelques indices sur les espaces de culture, notamment des jardins où sont produites les autres catégories de plantes. Le jardin médiéval catalan (hort) se présente sous la forme d'une petite parcelle consacrée à une culture de légumes et d'aromates, mais dans laquelle on trouve également des arbres fruitiers et des plantes textiles telles que le chanvre et le lin (Puig 2003). De la même façon, les cultures fruitières, surtout les vignes, sont réparties dans diverses parcelles selon le système du complant (association d'espèces dans la même parcelle) (Puig 2003; Durand et Puig, à paraître). L'approche par les sources carpologiques montre ses limites pour ce groupe de productions important dans l'économie agraire et vivrière puisque la simple attestation des fruits ne prouve pas la culture locale de l'arbre. Mais ils renseignent sur ceux que les habitants consommaient. Ainsi, les niveaux d'activité de la fin du $\mathrm{XI}^{\mathrm{e}}$ au début XIII ${ }^{\mathrm{e}}$ siècle du castrum montagnard des Angles ont montré que les occupants ont eu accès à des amandes, des mûres noires ou des figues. Ces denrées obtenues en dehors du terroir local ont pu être achetées sur les marchés comme celui de Puigcerda qui proposaient des fruits et des légumes apportés par les paysans des vallées voisines : pêches, poires, ail ou oignon (Rendu 1991, 88). Les mûres noires pourraient ainsi provenir des cultures signalées en 1022 à Saint-Just de Cerc (Seu de Urgell) ${ }^{36}$ ou en 1024 à Moligt (Conflent, Pyrénées-Orientales).

Les textes sont ainsi bien plus informatifs sur les lieux de production des fruitiers dans la région : olivier, figuier, noyer et chêne ont fait l'objet de cultures en Roussillon. Toutefois, ils apparaissent aussi très lacunaires. En effet, la mention d'autres fruitiers dans les toponymes médiévaux confirme qu'ils sont plus présents dans le paysage médiéval que ne l'indiquent les

\footnotetext{
${ }^{35}$ ADPO, B 140 fol. $129 \mathrm{v}^{\circ}$.

${ }^{36}$ « in ipso morario super ipsa casa medietate» (Urgellia $4 \mathrm{n}^{\circ} 375$ ) Nous remercions E. Bille de nous avoir signalé cette attestation.
} 
sources écrites. Ces arbres fruitiers ne constituraient pas un enjeu majeur méritant de figurer dans la documentation.

\section{Importation et exportation : le commerce des denrées}

L'importation des denrées exotiques : dattes, agrumes, riz

L'importation depuis la péninsule ibérique ou les pays méditerranéens d'espèces exotiques est documentée par les textes et permet de suivre les circuits commerciaux. Pour la période médiévale, les contextes archéologiques pyrénéens, en majorité ruraux, n'en livrent aucune trace. La datte n'est indiquée qu'à Collioure et témoigne d'un commerce entre le Languedoc et le royaume de Majorque. Mais l'origine des fruits est difficile à identifier ; ils pourraient provenir de la région d'Elche, mais surtout du Maghreb. Les agrumes sont indiqués dans la deuxième leude de Collioure (1297) et apparaissent comme des produits nouveaux, récemment introduits sur les marchés roussillonnais. Exceptionnellement utilisés dans les plats des recettes culinaires septentrionaux, les agrumes sont bien présents dans les réceptaires catalans et languedociens : jus de citron ou d'orange amère servent à confectionner des sauces qui accompagnent le poisson frit (Mane 2006). Les carnets de voyage du médecin Jérôme Munzer, lors de son périple en Europe à la fin du $X^{\mathrm{e}}$ siècle, signalent la présence d'orangers dans les jardins de son hôte à Perpignan (Tarayre 2006). Le riz, en revanche, seule céréale identifiée importée, est signalé dans les deux leudes.

L'ensemble de ces observations confirme l'existence d'un commerce à grande échelle de produits exotiques (fruits et céréales) depuis le sud de la Catalogne et témoigne de toute évidence d'une curiosité de goût de la part des Roussillonnais. Il est ensuite difficile d'évaluer la part des produits achetés et consommés localement et la part exportée vers le royaume de France et au-delà (Puig 2006).

\section{L'importation de denrées pourtant produites localement : blé, figues, raisins}

D'après les textes, on constate qu'en parallèle d'une production locale que montrent bien les découvertes carpologiques, certains grains ou fruits sont aussi importés. On prendra pour exemple le froment, placé derrière l'orge vêtue avec lequel il forme le duo céréalier de base d'après les attestations carpologiques. Au XIV ${ }^{\mathrm{e}}$ siècle, le Roussillon, comme tout le reste de la Catalogne, reçoit du blé de Sicile et de Tunis. Les textes font pourtant état à cette époque d'une abondance de blé dans cette région. Cette céréale se développe au début du XIV siècle, mais c'est l'orge qui, à cette période, alimente la plus grande partie de la population. Les arrivages de blé, à cette date, ne constituent pas, comme cela semble être le cas dans le reste 
de la Catalogne, une nécessité en période de disette, mais répondent plutôt à une demande accrue en une céréale jusque-là réservée aux nantis (Puig 2003).

La figue est un autre exemple illustrant parfaitement ces importations de denrées pouvant être produites localement. Bien que l'identification de pépins de figues soit courante en France méridionale (Ruas 2005 ; Ruas et al. 2006) et, notamment, dans les échantillons nord-catalans (Ros 2010, 2013), la faible abondance numérique de ses restes liée à la nature des contextes de découvertes (absence de dépotoirs gorgés d'eau en Roussillon) ne permet pas d'évaluer l'importance de son rôle alimentaire dans cette zone. Le figuier peut être rencontré à l'état spontané et cultivé en Roussillon (Gautier 1898) comme en Languedoc. Sa présence dans les assemblages carpologiques d'établissements ruraux est le plus souvent liée aux rejets domestiques et aux résidus de consommation. Une provenance locale de ces fruits, cultivés ou exploités en plaine, est attestée bien qu'elle ne soit que timidement confirmée par la documentation écrite ${ }^{37}$. Pourtant, lorsque la figue est citée dans les leudaires de Collioure, il s'agit toujours de fruits importés de la côte orientale de Péninsule ibérique, de toute évidence sous la forme de fruits séchés. Elles remontent ensuite vers le nord soit par cabotage, soit par voie terrestre. Curieusement, c'est le seul produit alimentaire indiqué dans la documentation écrite dont l'origine soit aussi variée. Ces documents signalent aussi que les taxes les plus élevées pèsent surtout sur les figues d'Alicante, de Tortose, Denia, de Malica et, éventuellement, Tarragone. Les figues de Mallorque, Valence et de Murcie sont moins taxées que les autres. L'origine géographique différencierait donc les produits selon une estimation dont on ignore les critères mais qui pourrait se rapprocher d'un label de qualité (Puig 2006). L'importation de ce fruit peut, en effet, recouvrir des raisons multiples telles que la recherche de variétés différentes (goût, forme, taille du fruit), d'une préparation spéciale (mode de séchage du fruit?), d'un lieu de production prestigieux, etc. Un phénomène similaire s'observe pour le raisin, qui, bien que cultivé dans la région roussillonnaise depuis le premier siècle de notre ère (Ros 2010, 2013), fait l'objet d'une importation sous forme de fruits secs, appelés « aztebib». Il s'agit de toute évidence d'un produit transformé, peut-être simplement séché, mais qui se distingue suffisamment du raisin roussillonnais pour porter une dénomination étrangère. Associé aux figues et aux dattes dans les leudaires, il emprunte très certainement les mêmes courants commerciaux qui sont déjà en place dans le milieu du XII siècle (Puig 2006).

\footnotetext{
${ }^{37}$ Comme nous l'avons vu plus haut, les arbres fruitiers apparaissent très peu dans la documentation écrite car ils ne sont pas taxés et suscitent donc peu l'intérêt des administrateurs (Puig 2003).
} 


\section{CONCLUSION}

La mise en relation des données textuelles et carpologiques a permis de dresser ici un premier état des connaissances sur les denrées végétales consommées et échangées en Roussillon entre le $\mathrm{X}^{\mathrm{e}}$ et le $\mathrm{XV}^{\mathrm{e}}$ siècle. Les résultats montrent l'existence d'une bonne diversité vivrière qui s'enrichit par l'importation d'espèces exotiques. À ce titre, on découvre que les importations concernent aussi des espèces pourtant produites en Roussillon (froment, figues, raisins), signe de la recherche de produits de qualités diverses et de besoins conjoncturels accrus.

La lecture croisée des données issues des assemblages carpologiques et des textes, présente bien souvent des difficultés inhérentes à la nature des sources, mais leur complémentarité permet d'éclairer certaines questions soulevées par l'absence ou la rareté des attestations ou le décalage régional. En effet, les données archivistiques médiévales apportent une vision de la production agricole strictement administrative. Toute la palette de végétaux cultivés et/ou cueillis échappe à l'inventaire lorsqu'ils ne sont pas taxés, que ce soit au moment de leur récolte ou de leur commerce. Ces données, bien que constituant un jalon, demandent à être étayées par l'augmentation du corpus carpologique. La nécessité de fouiller et d'échantillonner des contextes urbains, des sites d'habitat privilégié, des contextes en eau (ex : puits) ou autorisant la minéralisation des restes (ex : latrines) s'avère donc essentielle.

Remerciements :

Les données carpologiques sur le Roussillon ont été acquises lors d'un contrat doctoral du Muséum national d'Histoire Naturelle (Paris) (2010-2013) et dans le cadre du projet GDR 3644 BIOARCHEODAT (resp. J.-D. Vigne UMR 7209 CNRS-Muséum).

Acronymes :

ACA : Arxiu de la Corona d'Arago

ADPO : Archives départementales des Pyrénées-Orientales

\section{Sources publiées et d'érudition :}

Alart (J.-B), Cartulaire roussillonnais manuscrit, Médiathèque de Perpignan, XIXe siècle, 43 volumes. 
Agusti (M.), Llibre dels secrets de agricultura, casa rùstica i pastoril, Barcelone, 1617, Barcelone, Alta Fulla, 1988.

Germain Mouynés (M.), Inventaire des archives communales antérieures à 1790, Emmanuel Caillard Imprimeur, Narbonne, 1871, Annexes de la Série AA, pp. 4 à 6.

Gual Camarena (M.), Vocabularie del comercio medieval. Collección de aranceles aduaneros de la Corona de Aragón (Siglos XIII - XIV), Publicaciones de la Excelentìssima Diputación Provincial, Tarragone, 1968.

Les coutumes de Perpignan, introduction de J. Massot-Reynier, Nîmes, C. Lacour, 2003.

\section{BIBLIOGRAPHIE}

Albertini 2013 : ALBERTINI (L.), Essor de l'agriculture en al-Andalus (Ibérie arabe) $X^{e}$ $X I V^{e}$ siècle. Performances des agronomes arabo-andalous, L'Harmattan, Paris, 2013, 358 p.

Alessandri 1995 : ALESSANDRI (P.), Ruscino, des Wisigoths aux francs, in : SENAC (Ph.), éd., Histoire et archéologie des terres catalanes au Moyen Age, PUP, Collection Etudes, Perpignan, 1995, p. 9-48.

Alonso 2005 : ALONSO (N.), Agriculture and food from the Roman to the Islamic Period in the North-East of the Iberian peninsula : archaeobotanical studies in the city of Lleida (Catalonia, Spain), Vegetation History and Archaeobotany 14, 2005, p. 341-361.

Bois 1927 : BOIS (D.), Les plantes alimentaires chez tous les peuples et à travers les âges, Histoire, utilisation, culture, Encyclopédie biologique, Editions Paul Lechevalier, Paris, 1927, $637 \mathrm{p}$.

Bonnassie 1975-1976 : BONNASSIE (P.), La Catalogne du milieu du Xe siècle à la fin du XIe siècle. Croissance et mutations d'une société, Toulouse, Presses Universitaires du Mirail, 2 tomes, 1975-1976 (thèse d'État soutenue en 1973). 
Bonnassie 1990 : BONNASSIE (P.), La Catalogne au tournant de l'an mil, Paris, Albin Michel, 1990, 497 p.

Bouby 1996 : BOUBY (L.), Vauvenargues - La Petite Citadelle (Bouches-du-Rhône). Rapport carpologique préliminaire, rapport dactylographié, 1996.

Bouby 2010: BOUBY (L.), Agriculture dans le bassin du Rhône du Bronze final à l'Antiquité. Agrobiodiversité, économie, cultures. Doctorat de l'EHESS, Toulouse, 2 vol. dactylographiés, 2010, 1440 p.

Bouby 2014 : BOUBY (L.), Agriculture dans le bassin du Rhône du Bronze final à l'Antiquité. Agrobiodiversité, économie, cultures, Archives d'Écologie Préhistorique, Toulouse, 2014, 335 p.

Braudel 1961: BRAUDEL (F.), Alimentation et catégories de l'histoire, Annales, Economies, Sociétés, Civilisations, $16^{\mathrm{e}}$ année, 1961, p. 723-728.

Buxó et Piqué 2008 : BUXÓ (R.), PIQUÉ (R.), Arqueobotanica. Los usos de las plantas en la peninsula Iberica, Edition Ariel Prehistoria, Barcelona, 2008, 272 p.

Campmajo et al. 2007 : CAMPMAJO (P.), CRABOL (D.), BILLE (E.), RAYNAUD (Cl.), RUAS (M.-P.), PARENT (G.), RENDU (C.), Un atelier de traitement du fer sur le site du Haut Moyen Âge de la Coume de Païrounell à Angoustrine (Pyrénées-Orientales). Premiers résultats, in : CATAFAU (A.), éd., Domitia, 8-9, CHRISM, Université de Perpignan, 2007, p. 153-156.

Cheyronnaud 2006 : CHEYRONNAUD (A.), Le riz au Moyen Âge: de la culture à la consommation, Etude historique sur la situation du riz dans le sud de la France du XIII ème $^{\text {a }}$ siècle à l'époque moderne, Master 1, Université de Provence (Aix-Marseille I), 2006, 118 p.

Durand 1991 : DURAND (A.), Paysages, terroirs et peuplement dans les campagnes du Bas-Languedoc (Xe-XIIe siècles), Thèse, Paris I, 1991, 2 vol. 
Durand et Puig à paraître : DURAND (A.), Puig (C.), Une pratique culturale médiévale méconnue : le complantage en France méridionale, à paraître.

Gautier 1898 : GAUTIER (G.), Catalogue raisonné de la Flore des Pyrénées-Orientales, publication de la société agricole, scientifique et littéraire des Pyrénées-Orientales, Perpignan, $551 \mathrm{p}$.

Grieco 1987 : GRIECO (A. J.), Classes sociales, nourritures et imaginaire alimentaire en Italie (XIV $V^{\text {ème }} X V^{\text {ème }}$ siècles), thèse de 3e cycle, EHESS-Paris, 1987 [s.n.]

Grieco 1996 : GRIECO (A. J.), Alimentation et classes sociales à la fin du Moyen Age et à la Renaissance, in : FLANDRIN (J.-L.), MONTANARI (M.), dir., Histoire de l'alimentation, Fayard, Paris, 1996, p.479-490.

Hallavant 2009: HALLAVANT (Ch.), Etude carpologique du Château Saint-Pierre de Fenouillet : Premières données carpologiques, in : MASO (D.), éd, Château Saint-Pierre de Fenouillet, DFS fouille programmée, SRA Languedoc-Roussillon, Montpellier, 2009.

Hallavant et Ruas 2007 : HALLAVANT (Ch.), RUAS (M.-P.), Alimentation médiévale en montagne pyrénéenne d'après les graines et les fruits archéologiques, in : BOETSCH (G.), HUBERT (A.), éds., Montagne et alimentation, actes de l'université d'été de Vallouise 3-8 juillet 2006, Collection Anthropologie des populations alpines, Editions Hautes-Alpes, 2007, p. $35-52$.

Hallavant et Ruas 2008 : HALLAVANT (Ch.), RUAS (M.-P.), Pratiques agraires et terroir de montagne : un regard archéobotanique sur Montaillou (Ariège) au XIII ${ }^{\text {ème }}$ siècle, Archéologie du Midi Médiéval, 26, Carcassonne, 2008, p. 93-129.

Haudricourt et Hédin réed 1987 : HAUDRICOURT (A.-G.), HÉDIN (L.), L'Homme et les plantes cultivées, Gallimard, Paris, 1943, rééd. Métailié, Paris, 1987, 281p.

Jacomet 2006 : JACOMET (S.), Identification of cereal remains from archaeological sites, $2^{\text {ème }}$ édition, Fascicule dactylogrphié, Archaeobotany laboratory IPAS, Basel university, Suisse, 2006, 59 p. 
Lambert 1992 : LAMBERT (C.), dir., Du manuscrit à la table. Essai sur la cuisine au Moyen Âge et répertoire des manuscrits médiévaux contenant des recettes culinaires, Champion-Slatkine, Les Presses de l’Université de Montréal, Paris-Montréal, 1992.

Laurioux 1983 : LAURIOUX (B.), De l'usage des épices dans l'alimentation médiévale, Médiévales, 5, 1983, p. 15-31.

Mane 2006 : MANE (P.), Les fruits dans les traités culinaires français (XIII ${ }^{\text {ème }}-X^{\text {ème }}$ siècle), Archéologie du Midi Médiéval, 23-24, Carcassonne, 2006, p. 129-144.

Marinval 1999 : MARINVAL (Ph.), Les graines et les fruits : la carpologie, in : FERDIERE (A.), dir., La botanique, Collection « Archéologiques », Errance, Paris, 1999, p. 105-137.

Maurizio 1932 : MAURIZIO (A.), Histoire de l'alimentation végétale depuis la Préhistoire jusqu'à nos jours, Texte traduit du polonais par F. Gidon, Payot, Paris, 1932, 663 p.

Puig 2003 : PUIG (C.), Les campagnes roussillonnaises au Moyen Age : dynamiques agricoles et paysagères entre le XII ème et la première moitié du XIV ème siècle, thèse de doctorat d'Histoire, Université de Toulouse-Le Mirail, 2003, 918 p.

Puig 2005a : PUIG (C.), Les capbreus du roi de Majorque, 1292-1294, de l'estime au cadastre en Europe (XIII ${ }^{\text {ème }}$ - XVIII ${ }^{\text {ème }}$ siècles), in : RIGAUDIERE (A.), dir., Actes du colloque de Bercy, juin 2005, 2005.

Puig 2005b : PUIG (C.), À l'origine des premières taxes douanières : les leudaires en Roussillon et en Cerdagne (XIII ${ }^{\text {ème}}$-milieu du XIV ${ }^{\text {ème }}$ siècle), Douanes, États et Frontières dans l'est des Pyrénées de l'Antiquité à nos jours, PUP-AHAD, Saint-Estève, 2005, p. 23-40.

Puig 2006 : PUIG (C.), La place des fruits en Méditerranée nord-occidentale à partir des actes de la pratique et des tarifs marchands (XII ${ }^{\text {ème }}$-Première moitié XIV ${ }^{\text {ème }}$ siècles), Archéologie du Midi Médiéval, 23-24, Carcassonne, 2006, p. 119-128. 
Puig 2012 : PUIG (C.), L'apport de l'étude du stockage à notre connaissance de la conjoncture alimentaire autour de 1300 (Catalogne, Roussillon, Languedoc), Séminaire de l'Ecole Française de Rome, février 2004, Les disettes dans la conjoncture de 1300 en Méditerranée occidentale, 2012, p. 159-177.

Rendu 1991 : RENDU (C.), Un aperçu de l'économie cerdane à la fin du XIII ${ }^{\text {ème }}$ siècle : draps, bétail et céréales sur le marché de Puigcerdà en 1280-1281, Quadems d'Estudis Cerdans, Ceretania, 1, 1991, p. 87-106.

Ros 2010 : ROS (J.), Evolution des productions agricoles et alimentaires du Roussillon, de l'empire romain à l'an mil, Master 2, Université Paul Valéry Montpellier III, 2010, 2 vol., 97 p. et $58 \mathrm{p}$.

Ros 2013 : ROS (J.), Agrobiodiversité, pastoralisme, terroirs et patrimoines vivriers en Roussillon de la Protohistoire au Moyen Âge : archéobotanique et ethnoarchéologie, Thèse Muséum national d'histoire naturelle, Paris, 2013, 3 vol., 600 p.

Ros 2014 : ROS (J.), Rapport de l'analyse carpologique : site médiéval de Taxo l'Orangeraie, Argelès-sur-mer (Pyrénées-Orientales), in : GUINAUDEAU (N.), dir, Le site de l'Orangeraie (commune d'Argelès-sur-Mer, Pyrénées-Orientales), ACTER, Rapport Final d'Opération Archéologique, fouille préventive décembre 2012-avril 2013, 2014, vol. 3, 436 p.

Ros et Ruas 2014 : ROS (J.), RUAS (M.-P.), Cultures et alimentation végétale à Ruscino : les données carpologiques, in : RÉBÉ (I.), RAYNAUD (Cl.), SÉNAC (Ph.), éds, Le premier Moyen Âge à Ruscino (Château-Roussillon, Perpignan, Pyrénées-Orientales) Entre Septimanie et al-Andalus (VIIe-IXe s.) Hommage à Rémy Marichal, Monographie d'Archéologie Méditerranéenne 35, Lattes, 2014, p. 173-178.

Ros et al. accepté : ROS (J.), RUAS (M.-P.) et LEMOINE (M.), Identifier les résidus vitivinicoles carbonisés : référentiels expérimentaux et application au site romain du Petit Clos, in : DIESTCH-SELlAMI (M.-F.), HALLAVANT (C.) et BOUBY (L.), eds., Actes des 10e rencontres d'Archéobotanique, Les Eyzies-de-tayac, Supplément à la revue Aquitania. 
Ruas 1989 : RUAS (M.-P.), Les paléo-semences carbonisées de la fosse 1 (Antiquité tardive), Analyse paléocarpologique, in : GUILAINE (J.), VAQUER (J.), COULAROU (J.) et TREINEN-CLAUSTRE (F.), éds., Ornaisons-Médor. Archéologie et écologie d'un site de l'Âge du Cuivre, de l'Âge du Bronze final et de l'Antiquité tardive, Toulouse, Centre d'Anthropologie des Sociétés Rurales, Carcassonne, Archéologie en Terre d'Aude, 1989, p. 247-261.

Ruas 1990 : RUAS (M.-P.), Analyse des paléo-semences carbonisées, in : RAYNAUD (Cl.), dir., Le village gallo-romain et médiéval de Lunel-Viel (Hérault). La fouille du quartier ouest (1981 - 1983), Centre de Recherches d'Histoire Ancienne, Annales Littéraires de Besançon, 97, 1990, p. 96-104.

Ruas 1998 : RUAS (M.-P.), Les plantes consommées au Moyen Âge en France méridionale d'après les semences archéologiques, in: CAMPECH. (S.), POUSTHOMIS-DALLE (N.) éds., Usages et goûts culinaires au Moyen Age en Languedoc et en Aquitaine, Actes du colloque (Carcassonne, 1996), Archéologie du Midi Médiéval, 15-16, 1998, p. 179-204.

Ruas 2002 : RUAS (M.-P.), Productions agricoles, stockage et finage en Montagne Noire médiévale, DAF 93, Paris, MSH, 2002, 231 p.

Ruas 2003 : RUAS (M.-P.), Des céréales et des fruits dans le niveau incendié de la cabane 81 à l'Orri d'En Corbill., in : RENDU (C.), dir., La Montagne d'Enveig. Une estive pyrénéenne dans la longue durée, Éditions Trabucaïre, Perpignan, 2003, p. 393-412.

Ruas 2005 : RUAS (M.-P.), Aspects of early medieval farming from sites in Mediterranean France, in : BUXÓ (R.), JACOMET (S.), BITTMAN (F.), éds, Interaction between Man and Plants. New Progress in Archaeobotanical Research, Actes du $13^{\text {ème }}$ Symposium de l'IWGP, Girona (Spain) may 2004, Vegetation History and Archaeobotany 14 (4), 2005, p. 400-415.

Ruas 2008: RUAS (M.-P.),Des graines et des pépins du $\mathrm{XIV}^{\mathrm{e}}-\mathrm{XVI}^{\mathrm{e}}$ siècle, in : DIEULAFAIT (F.) dir., Enquêtes en sous-sol ... En quête du Passé, catalogue de l'exposition, Montauban 04/01au 31/03/2008, Municipalité de Montauban, 2008, p. 122.

Ruas 2009 : RUAS (M.-P.), Étude carpologique, Tableaux du rapport sur le site des Baguères 56, in : KOTARBA (J.), éd, Diagnostic de la commune de Ponteilla, - Ponteilla (Pyrénées- 
Orientales), Rapport final d'opération de diagnostic, LGV66 - Liaison ferroviaire PerpignanLe Perthus, volume 4, INRAP Méditerranée, SRA Languedoc-Roussillon, Montpellier, 2009, p. 78-79.

Ruas 2010-2011 : RUAS (M.-P.), Rapport des analyses carpologiques des sites Camps de la Ribera 42 et Les Bagueres 56 (commune de Ponteilla), de Manresa (commune de Canohès) (Pyrénées-Orientales), in: KOTARBA (J.), éd, Vestiges ruraux à la périphérie d'un habitat du haut Moyen Âge, tome 1 - Rapport final d'opération Fouille archéologique, LGV66 Liaison ferroviaire Perpignan-Le Perthus, volume 11, INRAP Méditerranée, SRA Languedoc-Roussillon, Montpellier, 2010-2011, p.179-219.

Ruas 2011 : RUAS (M.-P.), Semences pour l'histoire des patrimoines végétaux cultivés et sauvages, des pratiques d'exploitation et de leurs espaces en France (1 ${ }^{e r}-18^{e}$ siècle), Habilitation à Diriger des Recherches, Université François Rabelais, Tours, 3 volumes, 2011, $667 \mathrm{p}$.

Ruas 2012 : RUAS (M.-P.), Étude carpologique des sites de Vignes de l'Espérance à Banyuls-dels-Aspres, in : JANDOT (C.) avec la coll. de MAZIERE (F.), RAUX (S.), éds, Deux habitats ruraux du IIe Age du Fer et du Moyen Âge restitués par leurs fosses, Rapport final d'opération, Pyrénées-Orientales, LGV 66, liaison ferroviaire Perpignan-Le Perthus, Inrap Méditerranéen, SRA Languedoc-Roussillon, Montpellier, 2012, p.58-75.

Ruas 2015 : RUAS (M.-P.), L'exploitation des ressources végétales - analyse carpologique, in: MOUTON (D.), dir., La Moutte d'Allemagne-en-Provence. Un castrum précoce du Moyen Âge provençal, Errance et Centre Camille Jullian, Bibliothèque d'Archéologie Méditerranéenne et Africaine, 19, 2015, p. 67-109.

Ruas et Rendu 2005 : RUAS (M.-P.), RENDU (C.) avec la coll. de BERGERET (A.), Glanes et cultures médiévales en haute montagne. Réflexions autour d'une cabane d'estive à Enveig et du château des Angles (Pyrénées-orientales), in : CATAFAU (A.), éd., actes du colloque international RESOPYR, Exploitation, gestion et appropriation des ressources montagnardes du Moyen Age aux Temps Modernes, 8-10 nov. 2002 Font-Romeu (PyrénéesOrientales), PUP, Perpignan, 2005, p. 147-184. 
Ruas et al. 2006 : RUAS (M.-P.), BOUBY (L.), PRADAT (B.), Les restes de fruits dans les dépôts archéologiques du Midi de la France $\left(\mathrm{V}^{\text {ème }}-\mathrm{XVI}^{\text {ème }}\right.$ siècle), Archéologie $d u$ Midi Médiéval 23-24, Carcassonne, 2006, p. 145-193.

Sigaut 2010 : SIGAUT (F.), Nomenclature et identification des produits, in : FRANCONIE (H.), CHASTANET (M.), SIGAUT (F.), éds., Couscous, boulgour et polenta. Transformer et consommer les céréales dans le monde, Karthala, Paris, 2010, p. 443-456.

Tarayre 2006 : TARAYRE (M.), Jérôme Munzer. Voyage en Espagne et au Portugal (14941495), Paris, Les Belles Lettres (collection La roue à livres), 2006, 250p.

Watson 1983 réed 2008 : WATSON (A.M.), Agricultural innovation in the early Islamic world, Cambridge Studies in Islamic Civilization, Cambridge University Press, Cambridge, $1983,284 \mathrm{p}$. 